\title{
Prevalência da hipertensão arterial na população portuguesa em contexto de férias e abordagem multivariada dos fatores de risco através do método HJ-Biplot: estudo piloto
} Ângela Pereira Serafim, ${ }^{1}$ Ana Luísa Martins-Ferreira, ${ }^{2}$ Maria Paula Serafim, ${ }^{3}$ Gonçalo Oliveira, ${ }^{4}$ Eleutério Pedro-Rocheta, ${ }^{2}$ Nelson Pires ${ }^{5}$

\section{RESUMO}

Objetivos: Avaliar a prevalência da hipertensão arterial (HTA) na população portuguesa em contexto de férias, assim como estudar a relação dos valores tensionais com fatores de risco através do método HJ-Biplot.

Tipo de estudo: Observacional, descritivo, do tipo transversal, com uma amostra não probabilística (por conveniência). Local: Vilamoura.

População: Amostra de ambos os sexos com idade igual ou superior a 18 anos.

Métodos: A avaliação das pressões arteriais (PA) foi realizada em Vilamoura, de 30 de julho a 5 agosto de 2017, em dois locais distintos: Praia da Falésia e Marina de Vilamoura. Foram avaliadas cerca de 1.000 pessoas, provenientes de diferentes regiões do país. A medição da PA, em mmHg, foi realizada segundo os procedimentos das Guidelines for the Management of Arterial Hypertension. Foi considerada HTA se pressão arterial sistólica (PAS) $\geq 140 \mathrm{mmHg}$ e/ou pressão arterial diastólica (PAD) $\geq 90 \mathrm{~mm}$ e/ou a fazer medicação anti-hipertensiva (das diferentes classes de anti-hipertensores). Foi aplicado um questionário em suporte informático com o objetivo de recolher parâmetros antropométricos, patologias associadas e hábitos e estilos de vida. Foi garantida a proteção e confidencialidade dos dados pessoais e de saúde.

Resultados: Os resultados mostraram que 33,3\% da população estudada tinha HTA. De entre os indivíduos com HTA, 80,4\% tinha conhecimento da sua situação de saúde e encontravam-se sob terapêutica médica anti-hipertensora, dos quais $68,6 \%$ apresentavam valores normais de TA. As diferenças observadas entre sexos e entre grupos etários na prevalência de HTA mostraram valores mais elevados no sexo masculino (M: $43,0 \%$ e F: $26,0 \%$ ) e na faixa etária > 65 anos (76,2\%). A interpretação multivariada (HJ-Biplot) permitiu reforçar o aumento da prevalência da HTA com alguns fatores de risco, nomeadamente o sexo, a idade, o Índice de Massa Corporal, comorbilidades (principalmente diabetes e dislipidemia) e estilo de vida, nomeadamente a atividade física e os hábitos tabágicos.

Conclusões: Os resultados do presente estudo sugerem, em comparação com outros estudos na população portuguesa e mundial, a existência de uma evolução positiva na redução da prevalência de HTA, acompanhada por um aumento do seu controlo, embora existam diferenças entre subgrupos populacionais, com fatores de risco inerentes.

Palavras-chave: Hipertensão arterial; Prevalência; População portuguesa; Análise multivariada; Fator de risco.

1. Universidade do Algarve, CIMA, Faculdade de Ciências e Tecnologia.

2. Hospital de Loulé. UCSP - Loulé.

3. Universidade do Algarve, Faculdade de Ciências Humanas e Sociais.

4. Universidade do Algarve. Departamento de Ciências Biomédicas e Medicina. Faculdade de Ciências e Tecnologia.

5. Jaba Recordati S.A. 


\section{INTRODUÇÃO}

A

OCDE refere que as doenças cardiovasculares são a principal causa de morte nos Estados-membro da União Europeia, representando cerca de $36 \%$ das mortes em 2010 . As doenças cardiovasculares abrangem diversas doenças relacionadas com o sistema circulatório, incluindo a doença isquémica cardíaca e as doenças cérebro-vasculares. Em Portugal, as doenças cardiovasculares atingiram uma percentagem de óbitos de 29,7\% em 2015 . $^{1}$

Diversos estudos epidemiológicos provaram a relevante associação da hipertensão arterial (HTA) à doença coronária, Acidente Vascular Cerebral (AVC) e insuficiência renal. ${ }^{2-5}$

A HTA é o principal e o mais frequente fator de risco cardiovascular modificável e o seu tratamento e controlo assumem importância central nas estratégias preventivas. ${ }^{5}$ Os custos associados à morbilidade e mortalidade resultantes do seu deficiente diagnóstico, a pouca efetividade na aplicação do tratamento e ainda a deficiente prevenção a nível populacional são um problema em todo o mundo e, principalmente, dos responsáveis pela saúde em cada país. ${ }^{1}$

A prevalência média de HTA na Europa é de 44\% (38-55\%), enquanto nos Estados Unidos da América (EUA) e Canadá se situa nos $30 \%$ e $27 \%$, respetivamente. Este excesso de prevalência de HTA na Europa é acompanhado de diferenças significativas na taxa de tratamento e controlo. Nos EUA e Canadá 23\% dos hipertensos estão controlados, proporção que desce para $8 \%$ (5-9\%) nos países europeus. ${ }^{4,6}$

Nos últimos 30 anos, a população portuguesa tem sido referida como tendo dos mais elevados níveis de tensão arterial média. ${ }^{7-8}$ Estima-se que a prevalência de HTA na população adulta portuguesa será de $42,1 \%$, estando apenas $39 \%$ dos doentes hipertensos medicados com fármacos anti-hipertensores e, destes, $28,9 \%$ estarão controlados. ${ }^{7,9}$

Em Portugal foram realizados vários estudos sobre a prevalência da HTA, nomeadamente o estudo PAP, ${ }^{10}$ onde foi bem demonstrada a realidade nacional no que diz respeito a todos os parâmetros de avaliação da HTA - mostrou uma prevalência da HTA na população portuguesa de $42,1 \%$. Os resultados do estudo PAP foram posteriormente complementados e parcialmente validados com outros estudos, como o VALSIM, ${ }^{11}$ que, em- bora com diferentes metodologias e objectivos, enriqueceram grandemente o nosso conhecimento sobre a HTA, apontando para valores de prevalência de $42,6 \%$. Posteriormente, o estudo PHYSA ${ }^{9}$ encontrou semelhante prevalência $(42,2 \%)$, embora com valores mais elevados para o conhecimento da situação de saúde, tratamento e controlo de HTA. Mais recentemente, o estudo INSEF ${ }^{12}$ mostrou uma tendência decrescente na prevalência da HTA na população portuguesa, com um valor de $36,0 \%$.

O conhecimento regular da realidade nacional no que respeita à evolução, tendência e situação atual das medidas epidemiológicas de frequência de HTA (incidência, prevalência e mortalidade) é fundamental para aprofundar a capacidade de controlo da HTA, em consonância com o preconizado no programa nacional para as doenças cérebro-vasculares ${ }^{1} \mathrm{e}$ para se poder saber da maior ou menor eficácia das estratégias estabelecidas, de forma a fundamentar novas estratégias de saúde e orientações aos seus profissionais.

Assim, será relevante a caracterização dos padrões tensionais mais frequentes em Portugal, identificando eventuais lacunas e alvos de atuação em termos de saúde pública, nomeadamente no que respeita à identificação de grupos de especial risco e também para a avaliação de programas de saúde de prevenção das doenças cardiovasculares.

Os objetivos principais do presente estudo foram avaliar a prevalência da HTA em Portugal, o conhecimento da situação de saúde e o nível de tratamento e de controlo numa amostra da população de ambos os sexos com idades iguais ou superiores a 18 anos. Foram objetivos secundários averiguar a eventual relação dos valores tensionais com fatores de risco: obesidade, tabagismo, alcoolismo, prática de exercício físico e história familiar de HTA, relacionando os dados obtidos e elaboração de grupos/caracterização desses mesmos grupos.

\section{MATERIAL E MÉTODOS}

Foi realizado um estudo observacional, descritivo, do tipo transversal com uma amostra da população portuguesa, em período de férias de verão.

A avaliação das pressões arteriais (PA) foi realizada em Vilamoura durante um período de sete dias (30 de julho a 5 agosto de 2017) em dois locais distintos: (i) Praia da Falésia, deVilamoura, das 9 às 19 horas; (ii) Ma- 
rina de Vilamoura, das 20 às 0 horas. Foram avaliadas cerca de 1.000 pessoas provenientes de diferentes regiões do país.

A medição da PA, em mmHg, segundo os procedimentos das Guidelines for the Management of Arterial Hypertension, ${ }^{13}$ foi realizada numa estrutura temporária, em ambiente calmo e assegurando a privacidade. Os valores da PA foram obtidos com um aparelho automático de braço OMRON M2 e braçadeiras adequadas, com preenchimento de mais de $3 / 4$ do comprimento do braço pela braçadeira, tendo-se efetuado três medições em repouso. As medições foram realizadas após 15 minutos de repouso na posição sentada com as costas apoiadas no encosto da cadeira e intervaladas com pelo menos cinco minutos. As medições foram efetuadas após a aplicação do questionário.

Foi considerada a presença HTA se pressão arterial sistólica (PAS) $\geq 140 \mathrm{mmHg}$ e/ou pressão arterial diastólica $(\mathrm{PAD}) \geq 90 \mathrm{~mm}$ e/ou a fazer medicação anti-hipertensiva das diferentes classes de anti-hipertensores: vasodilatadores, agentes de ação central, bloqueadores adrenérgicos alfa e/ou beta, inibidores do enzima conversor da angiotensina (IECA), bloqueadores do recetor do tipo 1 da angiotensina (ARA), inibidores da renina, diuréticos tiazídicos, diuréticos de ansa, diuréticos poupadores de potássio (eg. amilorida) e antagonistas da aldosterona (eg. espirololactona), bloqueadores do canal de cálcio.

Foi aplicado um questionário em suporte informático (tablets) com o objetivo de conhecer, entre outras informações, dados pessoais e parâmetros antropométricos (idade, sexo, peso e altura), patologias associadas (hipertensão arterial, problemas cardíacos, problemas no metabolismo dos lípidos, diabetes, hipertrofia benigna da próstata, problemas renais, hiperuricemia e astenia funcional) e hábitos e estilos de vida (hábitos tabágicos, consumo de álcool e prática de exercício físico).

Este estudo foi aprovado pela Comissão de Ética da Administração Regional de Saúde do Centro e todas as informações dos participantes foram codificadas para manter a confidencialidade dos dados pessoais e de saúde.

Apresenta-se, de seguida, a definição das variáveis estudadas, de acordo com a Comissão de Classificação Internacional de Cuidados Primários. ${ }^{14-15}$

Problemas cardíacos: diagnóstico de enfarte agudo do miocárdio ou doença cardíaca isquémica (com ou sem angina) ou arritmia cardíaca (fibrilhação auricular/flutter, auricular ou taquicardia paroxística) ou insuficiência cardíaca.

Doença cérebro-vascular: diagnóstico de AVC/ / trombose ou isquemia cerebral transitória ou doença vascular cerebral.

Problemas de lípidos: nível anormal de lipoproteínas ou hiperlipidemia ou nível elevado de colesterol e/ou triglicéridos e xantoma.

Diabetes: diabetes insulinodependentes ou diabetes não insulinodependentes.

Hipertrofia benigna da próstata: hiperplasia benigna da próstata ou barra mediana prostática ou obstrução da próstata ou prostatomegalia.

Problemas renais: glomerulonefrite aguda, nefropatia por analgésico ou glomerulonefrite crónica ou nefrite ou nefroesclerose ou síndroma nefrótico ou insuficiência renal ou proteinúria ou uremia.

Hiperuricemia: valores analíticos de ácido úrico no sangue acima do limite da normalidade ou a fazer terapêutica direcionada.

Astenia funcional: sensação generalizada de debilidade e falta de vitalidade, falta de força, que se sente tanto a nível físico como intelectual e que afeta a capacidade de trabalhar ou realizar tarefas simples e que não melhora com o descanso.

Hábitos tabágicos: problema de fumar, perturbação devida ao consumo de tabaco que leva a intoxicação aguda, uso nocivo com prejuízo para a saúde clinicamente importante, síndroma de dependência e estado de privação.

Hábitos alcoólicos: perturbação devida ao consumo de álcool que leva a intoxicação aguda com ou sem uma história de abuso crónico.

Prática de exercício físico: pelo menos 150 minutos de atividade física de intensidade moderada por semana ou 75 minutos de atividade vigorosa por semana.

Foram ainda calculados: o índice de massa corporal (IMC) pela fórmula IMC = peso $(\mathrm{Kg}) /$ altura $^{2}\left(\mathrm{~m}^{2}\right)$; a pressão arterial média (PAM) pela equação PAM = PAD + $1 / 3 *$ (PAS-PAD), na qual PAD equivale à diastólica e PAS equivale à sistólica; e a quantificação da exposição ao fumo do tabaco, a qual foi quantificada e expressa em UMA (Unidades Maço Ano = $\left(\mathrm{n}^{\circ}\right.$ cigarros fumados por dia/20) x no anos). Foi também considerada uma unidade de bebida padrão o que corresponde a 10-12 gramas de álcool por copo. ${ }^{15}$ 


\section{Análise dos dados}

Das cerca de 1.000 pessoas examinadas, só foram incluídas para análise as pessoas com os questionários completos e com idades $\geq$ a 18 anos, perfazendo um total de 843 pessoas analisadas. Assim, existe um erro de precisão de $3 \%$ a nível nacional, assumindo uma prevalência teórica de hipertensão de cerca de $30 \%$ (o valor aproximado e estimado em alguns países da Europa) e um nível de confiança de $95 \%$.

A análise estatística foi efetuada no programa SPSS $®$ Statistics, v. 21 e Microsoft® Excel 2010. Primeiramente foi realizada uma análise estatística descritiva. Para as variáveis categóricas (nominais e ordinais) foram determinadas as frequências absolutas e relativas; para as variáveis numéricas determinaram-se as medidas de localização central e de dispersão. Foi depois calculada a frequência absoluta e a prevalência de complicações entre os indivíduos da amostra.

As correlações entre pressões arteriais (sistólica e diastólica) e as variáveis antropométricas foram estudadas através do coeficiente de correlação de Spearman. Foi considerado um $p<0,01$ a fim das análises serem consideradas estatisticamente significativas.

Para a comparação dos valores tensionais dos diferentes grupos foi utilizada a análise de variância (ANOVA) e o post hoc teste Tukey, com um nível de significância inferior a 5\%.

Foi aplicada uma análise multivariada através do método HJ-Biplot, a qual permitiu relacionar todos os dados obtidos. Os métodos Biplot ${ }^{16-17}$ são técnicas de inspeção de dados multivariados, os quais permitem detetar grupos onde se identificam, através de gradientes, não só as tendências como as similaridades/dissimilaridades entre diferentes grupos da amostra estudada. Para tal foi utilizado o software MULTBIPLOT (MULTivariate Analysis using BIPLOT) nos dados transformados. A análise dos clusters (grupos) foi efetuada utilizando a distância Euclidiana através do HJ-BIPLOT.

\section{RESULTADOS}

\section{Caracterização da população}

A população estudada é composta de 480 mulheres (56,9\%) e 363 homens (43,1\%). As características desta população estão resumidas no Quadro I.

No grupo estudado, a faixa etária mais representada em ambos os sexos encontra-se entre os 35-64 anos, com uma percentagem superior a 50\%. Relativamente à distribuição da população pelas diferentes regiões de Portugal verifica-se que a região de Lisboa e Vale do Tejo (LVT) é a mais prevalente, seguida da região Norte, Centro, Algarve, Alentejo e ilhas. De referir que a população estudada inclui cerca de $6 \%$ de portugueses a residir no estrangeiro.

Quantos aos dados antropométricos analisados verifica-se que a altura, o peso e IMC apresentaram médias maiores entre os homens.

Relativamente às patologias diagnosticadas inquiridas verifica-se que $26,8 \%$ da população estudada apresenta HTA, sendo também observada alteração do metabolismo dos lípidos na mesma ordem de grandeza. Outros problemas de saúde, como problemas cardíacos, diabetes, hiperplasia benigna da próstata (HBP), problemas renais, ácido úrico elevado e astenia funcional, apresentam uma percentagem que varia de 4 a $12 \%$.

Na população estudada os hábitos e estilos de vida mostram que $16,3 \%$ da população estudada fuma, sendo que no sexo masculino a percentagem é superior $(19,8 \%)$ relativamente ao sexo feminino $(13,5 \%)$. Também a maioria da população consome álcool (61,8\%); no entanto, não foram registados consumos excessivos de álcool (superiores a 21 unidades de álcool por semana para homens e a 14 unidades de álcool nas mulheres). ${ }^{18}$

\section{Pressão arterial}

No Quadro II encontram-se as medidas de tendência central e de dispersão das pressões arteriais, tanto a sistólica quanto a diastólica, assim como da pressão arterial média e frequência cardíaca (FC). As médias das pressões arteriais (PAS, PAD e PAM) foram mais altas nos homens, contrariamente à FC, a qual foi superior no sexo feminino.

Na Figura 1-A encontram-se as pressões arteriais médias, tanto a sistólica quanto a diastólica, em cada faixa etária e em ambos os sexos. Verifica-se que a PAS e a PAD são significativamente superiores no sexo masculino relativamente ao sexo feminino, nas três faixas etárias consideradas $(p<0,05)$. Por outro lado, observou-se um aumento significativo, em ambos os sexos, dos valores de PAS e PAD da faixa etária < 35 anos para a faixa etária dos 35-64 anos; após este aumento, os valores tensionais sistólicos continuaram a progredir signifi- 


\begin{tabular}{|c|c|c|c|}
\hline & Sexo feminino & Sexo masculino & TOTAL \\
\hline & $480(56,9 \%)$ & $363(43,1 \%)$ & 843 \\
\hline Idade & $n(\%)$ & $n(\%)$ & $n(\%)$ \\
\hline$<35$ anos & $111(23,1 \%)$ & $80(22,0 \%)$ & $191(22,7 \%)$ \\
\hline 35-64 anos & $281(58,5 \%)$ & $220(60,6 \%)$ & $501(59,4 \%)$ \\
\hline$\geq 65$ anos & $88(18,3 \%)$ & $68(17,4 \%)$ & $151(17,9 \%)$ \\
\hline Região & $n(\%)$ & $n(\%)$ & $n(\%)$ \\
\hline Norte & $134(27,9 \%)$ & $112(30,9 \%)$ & $246(29,2 \%)$ \\
\hline Centro & $57(11,9 \%)$ & $44(12,1 \%)$ & $101(12,0 \%)$ \\
\hline Lisboa e Vale do Tejo (LVT) & $193(40,2 \%)$ & $127(35,0 \%)$ & $320(38,0 \%)$ \\
\hline Alentejo & $17(3,5 \%)$ & $8(2,2 \%)$ & $25(3,0 \%)$ \\
\hline Algarve & $50(10,4 \%)$ & $49(13,5 \%)$ & $99(11,7 \%)$ \\
\hline Ilhas & $2(0,4 \%)$ & $1(0,3 \%)$ & $3(0,4 \%)$ \\
\hline Emigrantes & $27(5,6 \%)$ & $22(6,1 \%)$ & $49(5,8 \%)$ \\
\hline Dados antropométricos & Média \pm SD (max-min) & Média \pm SD (max-min) & Média \pm SD (max-min) \\
\hline Altura $(\mathrm{cm})$ & $162,1 \pm 6,7(184-143)$ & $173,7 \pm 7,4(202-146)$ & $167,1 \pm 9,0(202-143)$ \\
\hline Peso (Kg) & $62,2 \pm 10,4(108-43)$ & $78,9 \pm 12,7(121-46)$ & $69,4 \pm 14,1(121-43)$ \\
\hline IMC (Kg/m2) & $23,7 \pm 4,0(42-16)$ & $26,1 \pm 3,7(39-17)$ & $24,7 \pm 4,0(42-16)$ \\
\hline Baixo peso $(\mathrm{IMC}<18,5)$ & $17,8 \pm 0,6(3,5 \%)$ & $17,8 \pm 0,5(0,8 \%)$ & $17,8 \pm 0,6(2,4 \%)$ \\
\hline Normal $(18,5<I M C<25,0)$ & $21,9 \pm 1,8(66,7 \%)$ & $23,0 \pm 1,4(42,1 \%)$ & $22,3 \pm 1,8(56,1 \%)$ \\
\hline Excesso de peso $(25,0<\mathrm{IMC}<30,0)$ & $26,9 \pm 1,4(22,5 \%)$ & $27,1 \pm 1,4(42,7 \%)$ & $27,0 \pm 1,4(31,2 \%)$ \\
\hline Obesidade (IMC > 30) & $33,3 \pm 3,0(7,3 \%)$ & $32,9 \pm 2,2(7,3 \%)$ & $33,0 \pm 2,6(10,3 \%)$ \\
\hline Patologias diagnosticadas & $n(\%)$ & $n(\%)$ & $n(\%)$ \\
\hline HTA & $102(21,3 \%)$ & $124(34,2 \%)$ & $226(26,8 \%)$ \\
\hline Problema cardíaco & $38(7,9 \%)$ & $35(9,6 \%)$ & $73(8,7 \%)$ \\
\hline Problemas no metabolismo dos lípidos & $134(27,9 \%)$ & $110(30,3 \%)$ & $244(28,9 \%)$ \\
\hline Diabetes & $20(4,2 \%)$ & $33(9,1 \%)$ & $53(6,3 \%)$ \\
\hline Hiperplasia benigna da próstata & - & $43(11,8 \%)$ & - \\
\hline Problemas renais & $8(1,7 \%)$ & $16(4,4 \%)$ & $24(2,8 \%)$ \\
\hline Ácido úrico elevado & $7(1,5 \%)$ & $27(7,4 \%)$ & $34(4,0 \%)$ \\
\hline Astenia funcional & $73(15,2 \%)$ & $31(8,5 \%)$ & $104(12,3 \%)$ \\
\hline Hábitos e estilo de vida & $n(\%)$ & $n(\%)$ & $n(\%)$ \\
\hline Tabágicos & $65(13,5 \%)$ & $72(19,8 \%)$ & $137(16,3 \%)$ \\
\hline Álcool & $174(44,2 \%)$ & $253(85,0 \%)$ & $427(61,8 \%)$ \\
\hline Exercício físico & $295(61,5 \%)$ & $230(63,4 \%)$ & $525(62,3 \%)$ \\
\hline
\end{tabular}

cativamente $(p<0,05)$, enquanto os diastólicos estabilizaram na faixa etária $\geq 65$ anos $(p>0,05)$.

Na Figura 1-B estão representadas a PAS e a PAD por região em ambos os sexos. Verifica-se que os valores medidos de PAS são aparentemente superiores no sexo masculino relativamente ao sexo feminino, em todas as regiões, apesar de essa diferença não ser significativa $(p>0,05)$, à exceção da região do Alentejo e na população de emigrantes, onde os valores de PAS são significativamente superiores no sexo masculino comparati- 
vamente com o feminino $(p<0,05)$. Este padrão de variação foi semelhante ao observado nos valores de PAD. Contudo, só na região do Alentejo é que se verifica diferença significativa entre sexos $(p<0,05)$. De referir que estes resultados devem ser vistos com alguma reserva devido à pouca representatividade do número de indivíduos nesta região.

Se considerarmos os sexos independentemente verifica-se que não existem diferenças significativas entre os valores de PAS e PAD nas diferentes regiões do país $(p>0,05)$.

\section{Prevalência de hipertensão arterial}

A prevalência de HTA estimada foi de $33,3 \%$, tendo sido mais elevada no grupo etário mais velho $(76,2 \%)$ e no sexo masculino (43,0\%) (Quadro III).

Do total de indivíduos com HTA 80,4\% tinha conhecimento da sua situação de saúde e referiu estar a tomar medicação anti-hipertensiva. Estas proporções foram mais elevadas no sexo feminino e aumentaram com a idade (Quadro III). Observou-se uma proporção mais elevada de indivíduos com conhecimento da sua situação de saúde e sob medicação anti-hipertensora na região de Lisboa e Vale do Tejo (LVT) e na população portuguesa emigrada (Quadro III).

Do total de indivíduos hipertensos sob tratamento médico 68,6\% apresentavam valores de PA dentro dos limites da normalidade, sendo esta proporção mais elevada no sexo feminino (76,5\%). Entre grupos etários verificou-se que, na faixa etária mais baixa ( $<35$ anos), $100 \%$ dos indivíduos com HTA sob medicação apresentavam valores de PA controladas. Por outro lado, consideradas as diferentes regiões verifica-se que a população emigrada e a população da região do Alentejo apresentam uma estimativa pontual mais elevada de controlo da tensão arterial (Quadro III).

A prevalência de HTA observada em cada faixa etária e em ambos os sexos encontra-se representada na Figura 2-A. Pela análise da figura verifica-se que a prevalência de HTA aumenta com a faixa etária, sendo su- perior no sexo masculino em todas as faixas etárias consideradas, atingindo valores máximos na faixa etária $>65$ anos, com percentagens de 68,2 e $84,1 \%$ nos sexos feminino e masculino, respetivamente.

Se se considerar a prevalência de HTA por região observam-se valores mais elevados de HTA na região do Alentejo e na região Centro (Quadro III). Verifica-se, mais uma vez, que a prevalência foi superior na população do sexo masculino em todas as regiões consideradas (Figura 2-B). De destacar nas regiões com maior HTA (Alentejo e Centro) prevalências de HTA de 62,5 e $52,3 \%$ no sexo masculino (Figura $2-\mathrm{B}$ ).

\section{Análise de correlação}

A PAS e a PAD, medidas na população feminina, correlacionam-se positivamente entre si $(r=0,675, p<0,01)$. Por outro lado, estas duas variáveis correlacionam-se inversamente com a altura e positivamente com idade, peso e IMC (Quadro IV). 


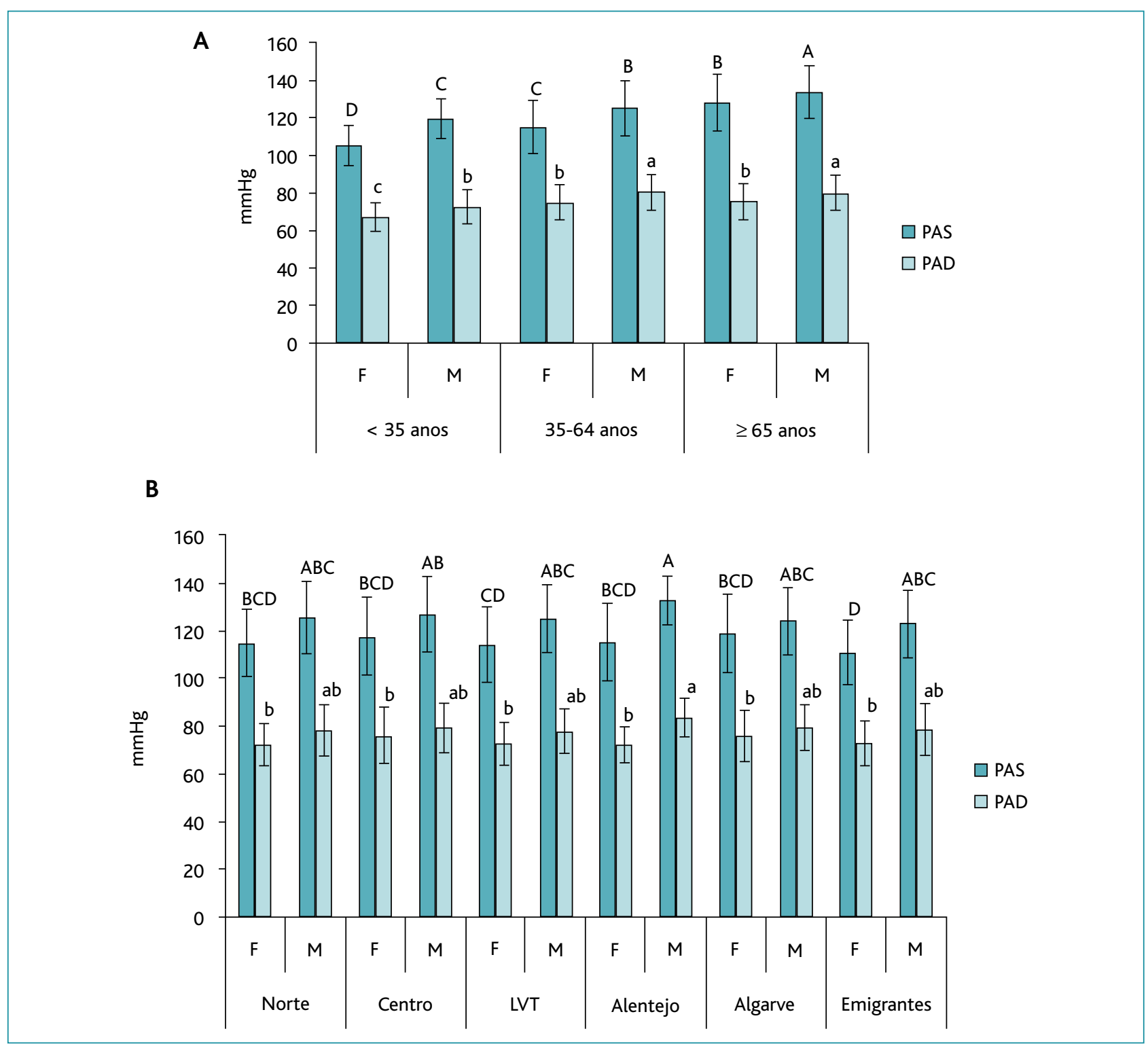

Figura 1. A) Valor médio de PAS e PAD em cada faixa etária e em ambos os sexos. B) Valor médio de PAS e PAD em cada região e em ambos os sexos.

Nota: Valores seguidos da mesma letra não são significativamente diferentes $(p>0,05)$.

A análise das correlações na população masculina mostrou, como acontece na população feminina, que os níveis tensionais (PAS e PAD) correlacionam-se entre si positivamente $(r=0,650, p<0,01)$ e com a idade, peso e IMC (Quadro V).

\section{Análise multivariada}

A aplicação de uma análise multivariada através do método HJ-Biplot permitiu relacionar todos os dados obtidos (respostas obtidas no questionário e medidas efetuadas de PA e FC). Esta análise foi efetuada separadamente em ambos os sexos. Nas Figura 3-A e Quadro VI encontram-se os resultados obtidos na população feminina, em que foi possível distinguir três grupos com características distintas.

O grupo ou cluster 1 corresponde ao grupo com o 
menor número de elementos (133) e diferencia-se dos outros grupos por conter uma população mais envelhecida $(62,5 \pm$ 9,9 anos), principalmente da região de Lisboa eVale do Tejo (43,6\%). Este grupo apresenta um valor médio de IMC indicativo de excesso de peso $\left(>25 \mathrm{Kg} / \mathrm{m}^{2}\right)$ e percentagens elevadas de patologias diagnosticadas, principalmente problemas no metabolismo dos lípidos (77\%) e hipertensão arterial (55\%). Relativamente aos hábitos e estilos de vida diferencia-se dos outros dois grupos por apresentar uma baixa percentagem de hábitos tabágicos $(6 \%)$. De referir que este grupo apresenta um valor médio de PAS mais elevada $(124 \mathrm{mmHg})$; no entanto, este valor não é significativamente diferente do grupo 2 (Figura 3-A, Quadro VI).

O grupo 2, com155 elementos, contém uma população feminina com uma média de idades de 48 anos, provenientes principalmente da região norte $(40,6 \%)$ e com um valor médio de IMC considerado normal $\left(23,8 \mathrm{Kg} / \mathrm{m}^{2}\right)$. Este grupo apresenta uma percentagem mais baixa de patologias associadas comparativamente ao grupo 1 (Figura 3-A, Quadro VI).

Por último, o grupo 3 contém o maior número de indivíduos ( $n=192)$ e apresenta características semelhantes ao grupo 2 , à exceção de incluir uma faixa etária mais nova (36,1 $\pm 14,4$ anos) e de serem principalmente da região LVT. Diferem ainda por apresentar hábitos tabágicos mais acentuados, maior consumo de álcool, mas também maior prática de exercício físico. Este grupo apresenta pressões artérias mais baixas (PAS de 102,3 $\pm 8,2 \mathrm{mmHg}$ e PAD de 66,5 $\pm 6,4 \mathrm{mmHg}$ ) comparativamente aos outros dois grupos (Figura 3-A, Quadro VI).

A aplicação de uma análise multivariada na população masculina mostrou, como aconteceu no sexo feminino, que a população estudada pode ser divida em três grupos com características distintas (Figura 3-B, Quadro VII).

Na população masculina, o grupo 1 e 2 apresentam características muito semelhantes, correspondendo aos grupos que incluem a população mais idosa, prin- cipalmente da região Norte, com um IMC indicativo de excesso de peso e com várias patologias diagnosticadas. Estes dois grupos apenas se diferenciam pelos hábitos e estilos de vida, em que os hábitos tabágicos e o consumo de álcool são mais acentuados no grupo 2. Estes dois grupos apresentam valores de PA superiores comparativamente ao grupo 3 (Figura 3-B, Quadro VII).

De facto, o grupo 3 corresponde ao grupo com o maior número de elementos (166) e diferencia-se dos outros grupos por conter uma população mais jovem (36,2 $\pm 13,3$ anos), principalmente da região Norte $(35,5 \%)$, com um IMC considerado normal $(24,3$ $\left.\mathrm{Kg} / \mathrm{m}^{2}\right)$, com baixas percentagens de patologias diagnosticadas, com hábitos tabágicos e prática de exercício físico. Como referido anteriormente, este grupo apresenta valores de PAS e PAD mais baixos (Figura 3-B, Quadro VII).

\section{DISCUSSÃO}

Em Portugal as doenças cardiovasculares são a primeira causa de morte, sendo a HTA um dos principais fatores de risco, muitas vezes subdiagnosticado ou subcontrolado. ${ }^{19}$ Assim, estudos que permitam aumentar 
A
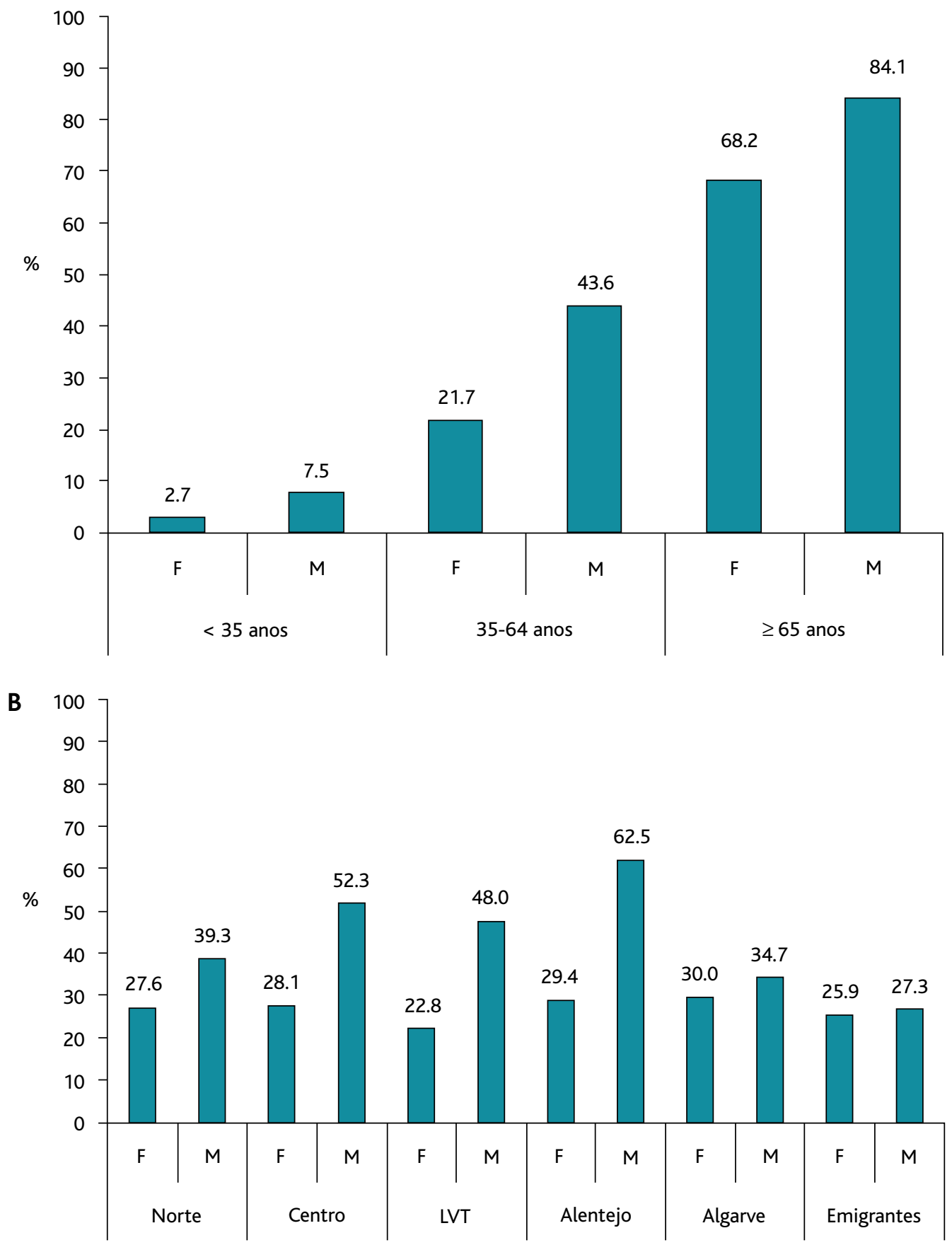

Figura 2. A) Prevalência da hipertensão arterial em cada faixa etária por sexo. B) Prevalência da hipertensão arterial por região em ambos os sexos. 
a compreensão sobre o estado do controlo da HTA na população portuguesa tornam-se essenciais para alertar a sociedade e a comunidade científica sobre este problema, pois os doentes com HTA não controlada têm risco acrescido de morte prematura, de eventos cerebrovasculares e de insuficiência cardíaca. ${ }^{20}$

No presente estudo, as observações clínicas foram independentes de consulta ou ida aos Centros de Saúde, realizadas no ambiente natural, em contexto de férias, com uma excelente taxa de participação de ambos os sexos e faixas etárias. Por outro lado, foi considerada uma abordagem multivariada no tratamento dos dados, que permite uma melhor interpretação dos fatores associados em cada grupo.

\section{Pressão arterial}

Os valores tensionais médios referentes à PA sistólica de $120 \pm 16 \mathrm{mmHg}$ (feminino: $115 \pm 15 \mathrm{mmHg}$ e masculino: $125 \pm 15 \mathrm{mmHg}$ ) e à PA diastólica de $76 \pm 10 \mathrm{mmHg}$ (feminino: $73 \pm 10 \mathrm{mmHg}$ e masculino: $79 \pm 10 \mathrm{mmHg}$ ) registados no presente estudo são inferiores aos documentados anteriormente na população portuguesa, nomeadamente no Estudo VALSIM (PAS: $136 \pm 19$

mmHg e PAD: $79 \pm 11 \mathrm{mmHg}{ }^{11}$ e no Estudo PAP (PAS: $135 \pm 21 \mathrm{mmHg}$ e PAD: $81 \pm 11 \mathrm{mmHg}$. ${ }^{7}$ De referir, no entanto, que não podemos excluir que estas diferenças possam estar relacionadas quer com as características das populações-alvo dos diferentes estudos, quer a nível metodológico que, como referido anteriormente, no presente estudo as observações clínicas são independentes de consulta médica.

Os valores tensionais foram globalmente mais elevados nos homens, sendo maior a diferença ao nível da PA sistólica, resultados coincidentes com os registados no Estudo VALSIM. ${ }^{11}$ Por outro lado, observou-se um aumento dos valores de PA sistólica e diastólica da faixa etária < 35 anos para a faixa etária dos 35-64 anos,

\begin{tabular}{|c|c|c|c|c|c|c|c|}
\hline \multicolumn{8}{|c|}{$\begin{array}{l}\text { QUADRO IV. Coeficientes de correlação de Spearman entre os valores de } \\
\text { pressões arteriais e frequência cardiaca com a idade e medidas } \\
\text { antropométricas, na população feminina }\end{array}$} \\
\hline & PAS & PAD & FC & Idade & Altura & Peso & IMC \\
\hline PAS & 1,000 & & & & & & \\
\hline PAD & $0,675^{*}$ & 1,000 & & & & & \\
\hline FC & $-0,013$ & 0,120 & 1,000 & & & & \\
\hline Idade & $0,514^{*}$ & $0,311^{*}$ & $-0,121$ & 1,000 & & & \\
\hline Altura & $-0,131 *$ & $-0,068$ & $-0,008$ & $-0,359 *$ & 1,000 & & \\
\hline Peso & $0,330^{*}$ & $0,251^{*}$ & $-0,009$ & $0,295^{*}$ & $0,277^{*}$ & 1,000 & \\
\hline IMC & $0,396^{*}$ & $0,281^{*}$ & $-0,008$ & $0,472^{*}$ & $-0,217^{*}$ & $0,876^{*}$ & 1,000 \\
\hline
\end{tabular}

* Significativo para $p<0,01$ (para $n=480, r$ é significativo para valores superiores a 0,128 ).

QUADRO V. Coeficientes de correlação de Spearman entre os valores de pressões arteriais e frequência cardíaca com a idade e medidas antropométricas, na população masculina

\begin{tabular}{l|c|c|c|c|c|c|c} 
& PAS & PAD & FC & Idade & Altura & Peso & IMC \\
\hline PAS & 1,000 & & & & & & \\
\hline PAD & $0,650^{*}$ & 1,000 & & & & & \\
\hline FC & 0,085 & 0,110 & 1,000 & & & & \\
\hline Idade & $0,362^{*}$ & $0,330^{*}$ & $-0,186^{*}$ & 1,000 & & & \\
\hline Altura & $-0,013$ & 0,004 & 0,024 & $-0,282^{*}$ & 1,000 & & \\
\hline Peso & $0,252^{*}$ & $0,267^{*}$ & $-0,012$ & $0,151^{*}$ & $0,495^{*}$ & 1,000 & \\
\hline IMC & $0,302^{*}$ & $0,301^{*}$ & $-0,024$ & $0,337^{*}$ & $-0,035$ & $0,848^{*}$ & 1,000 \\
\hline
\end{tabular}

* Significativo para $p<0,01$ (para $n=363, r$ é significativo para valores superiores a 0,148 ).

após o que os valores tensionais sistólicos continuaram a progredir, enquanto os diastólicos estabilizaram na faixa etária $\geq 65$ anos. Esta progressão dos valores tensionais com a idade também foi encontrada no estudo VALSIM ${ }^{11}$ e estão de acordo os resultados de Rocha $^{21}$ e Lopes, ${ }^{22}$ que defendem que, a partir da meia-idade, a PAD tende a diminuir enquanto a PAS tende a aumentar.

\section{Hipertensão arterial}

Os resultados obtidos mostram que 33,3\% da população estudada tinha HTA. De entre os indivíduos com HTA estima-se que $80,4 \%$ tivesse conhecimento da sua situação de saúde e que a mesma percentagem esti- 


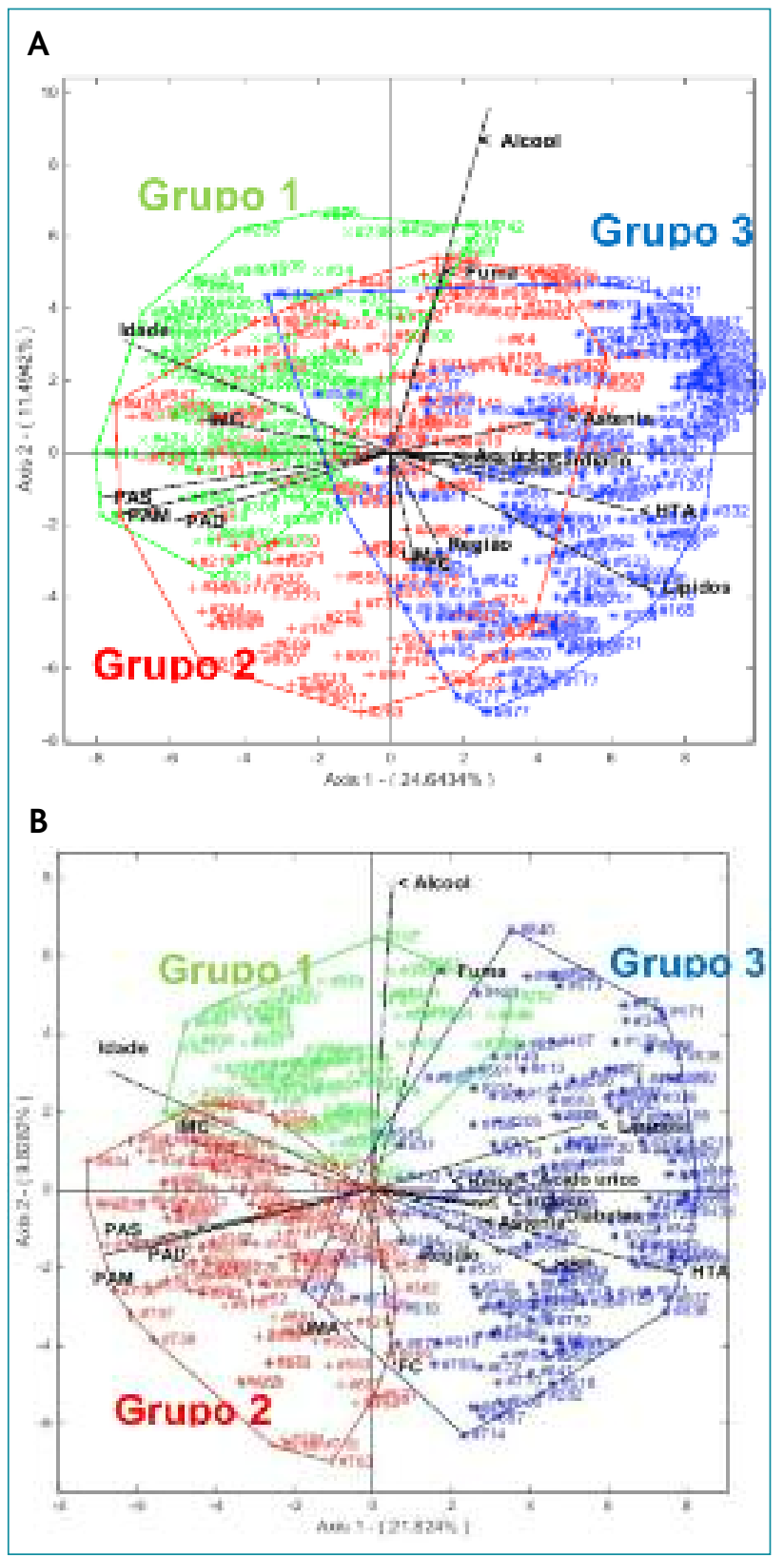

Figura 3. Análise multivariada através do método HJ-Biplot: A) população feminina. B) população masculina.

vesse sob terapêutica médica anti-hipertensora, sendo que, destes, $68,6 \%$ apresentava valores normais de PA (Quadro III).

Em relação a estudos anteriores sobre a prevalência da HTA na população portuguesa, como nos estudos mais recentes que apontam para uma tendência de- crescente, o valor da prevalência da HTA estimado no presente estudo mostra essa diminuição [estudo PAP, 2003: 42,1\%; ${ }^{7}$ estudo VALSIM, 2006-07: 42,62\%; ${ }^{11}$ estudo PHYSA, 2011-12: 42,2\%; ${ }^{9}$ estudo em 2013: 29,1\%; ${ }^{23}$ estudo INSEF, 2015: 36,0\% ${ }^{12}$ ]. Também a proporção de indivíduos com HTA que refere ter conhecimento da sua situação de saúde e que se encontram sob tratamento $(80,4 \%)$ é superior ao observado em outros estudos, nomeadamente em $2003^{7}(45,7 \%)$, em 2011-2012 $(76,6 \%)$ e em $2015^{12}$ (69,8\%). Diferenças no mesmo sentido foram encontradas no que se refere à proporção de indivíduos sob tratamento [PAP, 2003: 38,9\%; ${ }^{7}$ PHYSA, 2011-2012: 74,9\%; ${ }^{9}$ INSEF, 2015: 69,4\%. ${ }^{12}$ De referir, mais uma vez, que não podemos excluir que estas diferenças possam estar relacionadas quer com as características das populações-alvo dos diferentes estudos, quer a nível metodológico.

Relativamente à taxa de controlo nos hipertensos tratados, o presente estudo estimou uma taxa de $68,6 \%$ no global da amostra, percentagem superior à reportada em Portugal em $2003(11,2 \%)^{7}, 2010(30,7 \%)^{24}, 2011$ $(47,7 \%)^{25}$ e 2011-2012 (42,5\%) ${ }^{9}$ e mais próxima da apontada em $2015(71,3 \%)^{12}$, o que sugere que o controlo da HTA, quando tratada, tem vindo a aumentar nos últimos anos. Esta evolução confirma-se com os dados obtidos por outros autores e deve-se a uma maior efetividade do tratamento anti-hipertensivo ao nível dos cuidados de saúde primários. ${ }^{23}$

As diferenças observadas entre sexos e entre grupos etários na prevalência de HTA estão de acordo com o descrito na literatura, ${ }^{9-10,26}$ em que foram observados valores mais elevados no sexo masculino e na faixa etária $>65$ anos (Quadro III). Contrariamente aos estudos anteriores, o estudo de Macedo e Ferreira, ${ }^{24}$ com dados de 2013, referentes a todos os utentes que tiveram pelo menos duas medições da pressão arterial registadas em todas as unidades de saúde existentes em Portugal, estimaram uma prevalência superior no sexo feminino $(31,8 \%)$ relativamente ao sexo masculino $(26 \%)$. Estas diferenças parecem mostrar, para além das diferenças biológicas, a importância de outros fatores de risco de hipertensão como, por exemplo, um consumo mais elevado de sal e de tabaco. ${ }^{27}$

Por outro lado, na população com menos de 35 anos a prevalência de HTA é baixa $(4,7 \%)$ e apresenta um bom controlo da HTA. Estes dados estão de acordo com 
o estudo de Macedo e Ferreira, ${ }^{24}$ que também refere valores estimados muito baixos de prevalência de HTA para esta faixa etária.

Se se considerar a prevalência no grupo etário 35-64 anos, a qual é mais utilizada na comparação das prevalências de HTA entre países, verifica-se que a estimada no presente estudo - 31,3\% (H: 43,6\%; M: 21,7\%) encontra-se na mesma ordem que outros países europeus, como a Suécia $(38 \%)^{28}$ e a Itália $(38 \%)^{4}$ e inferiores aos registados em Inglaterra $(42 \%),{ }^{29}$ Espanha $(47 \%)^{30} \mathrm{e}$ Alemanha $(55 \%)$. $^{4}$ Por outro lado, é superior quando comparada com os valores estimados nos Estados Unidos da América $(29,5 \%)^{6}$ e no Canadá (27\%). ${ }^{31}$

No que se refere aos indicadores regionais verificou-se que a prevalência de HTA exibiu variação entre as várias regiões. Em Portugal Continental detetou-se a prevalência mais elevada na região do Alentejo e na região Centro e mais reduzida na região Norte (Quadro III). Estes resultados que estão em parte em concordância com os determinados por Macedo e colaboradores, ${ }^{7}$ Cortez-Dias e colaboradores ${ }^{11}$ e por Macedo e Ferreira, ${ }^{24}$ os quais mostraram uma prevalência superior no Alentejo e inferior na região Norte.

Um resultado que merece especial atenção é a prevalência de HTA estimada para a população emigrada, por ser o grupo com menor prevalência $(26,5 \%)$. Este grupo é também aquele com a maior proporção de indivíduos tratados e controlados (Quadro III).

\section{Análise multivariada}

A aplicação de uma análise multivariada através do método HJ-Biplot permitiu distinguir três grupos com características distintas, em ambos os sexos.

A interpretação dos gráficos HJ-Biplot permitiu reforçar os fatores de risco assumidos por outros autores no aumento da tensão arterial, nomeadamente o sexo, a idade, o índice de massa corporal, comorbilidades (principalmente diabetes mellitus e dislipidemia), os estilos de vida, nomeadamente a atividade física e os hábitos tabágicos. ${ }^{32-33}$ De facto, estudos prévios confirmaram uma correlação significativa dose-efeito entre 


\begin{tabular}{|c|c|c|c|}
\hline & Cluster 1 & Cluster 2 & Cluster 3 \\
\hline & $70(19,3 \%)$ & $127(35,0 \%)$ & $166(45,7 \%)$ \\
\hline Idade (média \pm SD) & $60,8 \pm 12,5$ & $57,7 \pm 11,4$ & $36,2 \pm 13,3$ \\
\hline Região moda* (\%) & $3(43,0 \%)$ & $3(36,2 \%)$ & $1(35,5 \%)$ \\
\hline \multicolumn{4}{|l|}{ Dados antropométricos (média \pm SD) } \\
\hline \multicolumn{4}{|l|}{ Patologias diagnosticadas $n(\%)$} \\
\hline HTA & $43(25,9 \%)$ & $78(47,0 \%)$ & $3(1,8 \%)$ \\
\hline Problema cardíaco & $10(6,0 \%)$ & $21(12,7 \%)$ & $4(2,4 \%)$ \\
\hline Problemas no metabolismo dos lípidos & $19(19,0 \%)$ & $76(45,8 \%)$ & $15(9,0 \%)$ \\
\hline Diabetes mellitus & $9(9,0 \%)$ & $22(13,3 \%)$ & $2(1,2 \%)$ \\
\hline Hiperplasia benigna da próstata & $22(13,3 \%)$ & $20(12,0 \%)$ & $1(0,6 \%)$ \\
\hline Problemas renais & $4(2,4 \%)$ & $11(6,6 \%)$ & $1(0,6 \%)$ \\
\hline Ácido úrico elevado & $7(4,2 \%)$ & $19(11,4 \%)$ & $1(0,6 \%)$ \\
\hline Astenia funcional & $9(5,4 \%)$ & $19(11,4 \%)$ & $3(1,8 \%)$ \\
\hline \multicolumn{4}{|l|}{ Hábitos e estilo de vida $n(\%)$} \\
\hline Tabágicos & $2(1,2 \%)$ & $39(23,5 \%)$ & $31(18,7 \%)$ \\
\hline Álcool & $18(10,8 \%)$ & $115(69,3 \%)$ & $120(72,3 \%)$ \\
\hline Exercício físico & $38(22,9 \%)$ & $59(35,5 \%)$ & $133(80,1 \%)$ \\
\hline \multicolumn{4}{|c|}{ Pressão arterial e frequência cardíaca (média \pm SD) } \\
\hline PAS (mmHg) & $126,6 \pm 11,5$ & $135,4 \pm 14,0$ & $117,2 \pm 10,7$ \\
\hline PAD (mmHg) & $77,7 \pm 8,3$ & $85,8 \pm 7,9$ & $73,5 \pm 8,4$ \\
\hline FC (bpm) & $69,1 \pm 10,6$ & $75,8 \pm 10,9$ & $75,6 \pm 13,4$ \\
\hline
\end{tabular}

*Moda 1 - Região Norte; moda 2 - Região Centro; moda 3 - Região de Lisboa e Vale do Tejo; moda 4 - Região do Alentejo; moda 5 - Região do Algarve; moda 6 - Região Autónoma dos Açores; moda 7 - Região Autónoma da Madeira; moda 8 - Emigrantes.

o número de cigarros fumados por dia e alterações dos

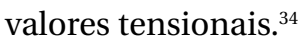

\section{Limitações}

Este estudo conta com algumas limitações metodológicas que devem ser consideradas na extrapolação dos resultados para a população em geral, nomeadamente no número limitado de amostras. Também é necessário considerar que a análise transversal não permite atribuir causalidades ou definir a direção de um efeito. Por outro lado, na utilização de uma amostragem não probabilística é necessário ter consciência dos possíveis vieses decorrentes de suas limitações.

De referir ainda que a população alvo foram pessoas

ção portuguesa. em contexto de férias, pelo que a extrapolação dos dados a nível nacional deve ter em conta que não foram incluídos todos os extratos sociais.

Outra limitação metodológica que deve ser tomada em consideração, é o facto da não existência de um processo de validação do questionário aplicado.

Seria importante incluir outras variáveis, como: grau de escolaridade, estado civil, nível socioeconómico, nível da utilização dos cuidados de saúde, adesão à medicação, padrão de tratamento, entre outros fatores já definidos noutros estudos portugueses associados ao controlo da HTA na popula-

Também é importante realçar que as medições efetuadas em indivíduo com valores de pressão arterial elevados, detetados num único registo temporal, não permitem assumir o diagnóstico de HTA. Por outro lado, o facto de as medições terem sido realizadas em diferentes períodos do dia torna obrigatório considerar-se a possível influência do ritmo circadiano nos valores tensionais.

\section{CONCLUSÃO}

Em conclusão, e apesar das limitações, os objetivos do presente estudo foram atingidos.

Os resultados sugerem, em comparação com outros 
estudos na população portuguesa e mundial, a existência de uma evolução positiva na redução da prevalência de HTA, acompanhada por um aumento do seu controlo, embora existam diferenças entre subgrupos populacionais, com fatores de risco inerentes.

\section{AGRADECIMENTOS}

A realização deste projeto contou com o apoio da JABA e a colaboração da Marina de Vilamoura.

\section{REFERÊNCIAS BIBLIOGRÁFICAS}

1. Direção-Geral da Saúde. Programa nacional para as doenças cérebrovasculares 2017. Lisboa: DGS; 2017.

2. KannelWB, Wolf PA, Verter J, McNamara PM. Epidemiologic assessment of the role of blood pressure in stroke: the Framingham Study. JAMA. 1996;276(15):1269-78.

3. KannelWB. Elevated systolic blood pressure as a cardiovascular risk factor. Am J Cardiol. 2000;85(2):251-5.

4. Wolf-Maier K, Cooper RS, Banegas JR, Giampaoli S, Hense HW, Joffres $M$, et al. Hypertension prevalence and blood pressure levels in 6 European countries, Canada, and the United States. JAMA. 2003;289(18): 2363-9.

5. Lim SS, Vos T, Flaxman AD, Danaei G, Shibuya K, Adair-Rohani H, et al. A comparative risk assessment of burden of disease and injury attributable to 67 risk factors and risk factor clusters in 21 regions, 19902010: a systematic analysis for the Global Burden of Disease Study 2010. Lancet. 2012;380(9859):2224-60.

6. Guo F, He D, ZhangW, Walton RG. Trends in prevalence, awareness, management, and control of hypertension among United States adults, 1999 to 2010. J Am Coll Cardiol. 2012;60(7):599-606.

7. Macedo ME, Lima MJ, Silva AO, Alcântara P, Ramalhinho V, Carmona J. Prevalence, awareness, treatment and control of hypertension in Portugal: the PAP study. Rev Port Cardiol. 2007;26(1):21-39.

8. Instituto Nacional de Estatística. Risco de morrer em 2012 [homepage]. Lisboa: INE; 2014. Available from: https://www.ine.pt/xportal/ xmain?xpid=INE\&xpgid=ine_publicacoes\&PUBLICACOESpub_boui=2 16382393\&PUBLICACOESmodo $=2 \& x$ lang $=p t$

9. Polónia J, Martins L, Pinto F, Nazare J. Prevalence, awareness, treatment and control of hypertension and salt intake in Portugal: changes over a decade - the PHYSA study. J Hypertens. 2014;32(6):1211-21.

10. Macedo ME, Lima MJ, Silva AO, Alcântara P, Ramalhinho V, Carmona J. Prevalence, awareness, treatment and control of hypertension in Portugal: the PAP study. J Hypertens. 2005;23(9):1661-6.

11. Cortez-Dias N, Martins S, Belo A, Fiuza M. Prevalence and management of hypertension in primary care in Portugal. Insights from the VALSIM study. Rev Port Cardiol. 2009;28(5):499-523.

12. Rodrigues AP, Gaio V, Kislaya I, Graff-Iversen S, Cordeiro E, Silva AC, et al. Prevalência de hipertensão arterial em Portugal: resultados do Primeiro Inquérito Nacional com Exame Físico (INSEF 2015) [Hypertension prevalence in Portugal: results from the first Portuguese Health Examination Survey 2015]. Observações Bol Epidemiol. 2017;6 Suppl 9:11-4. Portuguese

13. Mancia G, Fagard R, Narkiewicz K, Redon J, Zanchetti A, Böhm M, et al.
2013 ESH/ESC Practice guidelines for the management of arterial hypertension. Blood Press. 2014;23(1):3-16.

14. Comissão de Classificações da WONCA. Classificação internacional de cuidados de saúde primários [Internet]. $2^{\text {a }}$ ed. Lisboa: Administração Central do Sistema de Saúde; 2016. Available from: http://www.acss. min-saude.pt/wp-content/uploads/2016/07/1_ICPC_2_4_4_VF.pdf

15. Babor TF, Higgins-Biddle JC, Saunders JB, Monteiro MG. AUDIT - the Alcohol Use Disorders Identification Test: guidelines for use in primary health care. 2nd ed. Geneva:WHO; 2001.

16. Vicente-Villardón JL. MULTBIPLOT: a package for multivariate analysis using biplots. Salamanca: Departamento de Estadística, Universidad de Salamanca; 2010.

17. Serafim A, Company R, Lopes B, Silva N, Castela E, Bebianno MJ, et al. Profile analysis of mothers susceptible to contaminant exposure in the Algarve region: application of the HJ-BIPLOT method. Biometric Lett. 2012;49(1):57-66.

18. Batty GD, Lewars H, Emslie C, Gale CR, Hunt K. Internationally recognized guidelines for 'sensible' alcohol consumption: is exceeding them actually detrimental to health and social circumstances? Evidence from a population-based cohort study. J Public Health. 2009;31(3):360-5.

19. Ramos E, Barros H. Prevalence of hypertension in 13-year-old adolescents in Porto, Portugal. Rev Port Cardiol. 2005;24(9):1075-87.

20. Mendes R, Themudo-Barata JL. Envelhecimento e pressão arterial [Aging and blood pressure]. Acta Med Port. 2008;21(2):193-8. Portuguese

21. Rocha E. Influência da pressão arterial sistólica e pressão arterial diastólica na repercussão nos órgãos alvo. Factores Risco. 2013;(28):16-9.

22. Lopes E, Alarcão V, Simões R, Fernandes $M$, Gómez V, Souto $D$, et al. Controlo da hipertensão arterial nos cuidados de saúde primários: uma comparação entre nativos Portugueses e imigrantes dos Países Africanos de Língua Oficial Portuguesa [Hypertension control at the primary health care: a comparison among Portuguese natives and Portuguese Speaking African Coutries immigrants].Acta Med Port. 2016;29(3):193204. Portuguese

23. Macedo ME, Ferreira RC. Hipertensão arterial nos cuidados de saúde primários em Portugal: contributo para o conhecimento epidemiológico da população em 2013. Factores Risco. 2015;(36):47-56.

24. Polónia J, Mesquita-Bastos J, Pessanha P, Bertoquini S, Martins L, Silva JA, et al. Global cardiovascular risk stratification of hypertensive patients followed in Portugal in primary care or in hospital care according to the 2007 ESH/ESC guidelines. Rev Port Cardiol. 2010;29(11): 1685-96.

25. Oliveira-Martins S, Oliveira T, Gomes JJ, Caramona M, Cabrita J. Factors associated with arterial hypertension in pharmacy users in Portugal. Rev Saúde Pública. 2011;45(1):136-44.

26. Tolonen H, Giampaoli S, Kuulasmaa K, Mindell JS, Männistö S, Dias CM, et al. Blood pressure profiles, and awareness and treatment of hypertension in Europe: results from the EHES Pilot Project. Public Health. 2016;135:135-9.

27. Perdigão C, Rocha E, Duarte JS, Santos A, Macedo A. Prevalence and distribution of the main cardiovascular risk factors in Portugal: the AMALIA study. Rev Port Cardiol. 2011;30(4):393-432.

28. Persson M, Carlberg B, Mjörndal T, Asplund K, Bohlin J, Lindholm L. 1999 WHO/ISH Guidelines applied to a 1999 MONICA sample from northern Sweden. J Hypertens. 2002;20(1):29-35. 
29. Primatesta P, Poulter NR. Improvement in hypertension management in England: results from the Health Survey for England 2003. J Hypertens. 2006;24(6):1187-92.

30. Banegas JR, Rodríguez-Artalejo F, de la Cruz Troca JJ, Guallar-Castillón $P$, del Rey Calero J. Blood pressure in Spain: distribution, awareness, control, and benefits of a reduction in average pressure. Hypertension. 1998;32(6):998-1002.

31. Joffres MR, Ghadirian P, Fodor JG, Petrasovits A, Chockalingam A, Hamet P. Awareness, treatment, and control of hypertension in Canada. Am J Hypertens. 1997;10(10 Pt 1):1097-102.

32. Booth JN 3rd, Li J, Zhang L, Chen L, Muntner P, Egan B. Trends in prehypertension and hypertension risk factors in US adults: 1999-2012. Hypertension. 2017;70(2):275-84.

33. Pinto IC, Martins D. Prevalence and risk factors of arterial hyperten- sion: a literature review. J Cardiovasc Med Ther. 2017;1(2):1-7.

34. Al-Safi SA. Does smoking affect blood pressure and heart rate? Eur J Cardiovasc Nurs. 2005;4(4):286-9.

\section{CONFLITO DE INTERESSES}

Os autores declaram não haver quaisquer conflitos de interesse.

\author{
ENDEREÇO PARA CORRESPONDÊNCIA \\ Ângela Pereira Serafim \\ E-mail: aserafim@ualg.pt \\ https://orcid.org/0000-0002-1165-0645
}

Recebido em 09-02-2018

Aceite para publicação em 29-10-2019

\section{ABSTRACT}

\section{PREVALENCE OF HYPERTENSION AMONG THE PORTUGUESE POPULATION IN A HOLIDAY CONTEXT AND MULTIVARIATE ANALYSIS APPROACH OF RISK FACTORS THROUGH HJ-BIPLOT METHOD: A PILOT STUDY}

Purpose: The aim of the present study was to evaluate the prevalence of hypertension among the Portuguese population in a holiday context, as well as the relationship between blood pressure (BP) and risk factors, through the HJ-Biplot method.

Type of study: Observational, descriptive, transversal-type, with a non-probabilistic sample (convenience). Local:Vilamoura.

Population: Sample of both genders ( $\geq 18$ years old).

Methods: BP was measured in Vilamoura, from $30^{\text {th }}$ July to $5^{\text {th }}$ August 2017, in two distinct places: Praia da Falésia and Marina de Vilamoura. Around 1,000 people were evaluated, from different regions of the country (Portugal). BP measures, in $\mathrm{mmHg}$, followed the protocol from Guidelines for the Management of Arterial Hypertension. Hypertension was defined as mean systolic BP (SBP) $\geq 140 \mathrm{mmHg}$ and/or diastolic BP (DBP) $\geq 90 \mathrm{mmHg}$ and/or use of antihypertensive medication if hypertension was known. A questionnaire was applied in informatic support with anthropometric parameters, associated pathologies, habits and life Styles. Protection and confidentiality of personal data were guaranteed.

Results: The prevalence of hypertension was $33.3 \%$ of the studied population. Of those, $80.4 \%$ were aware of their health state and they were under antihypertensive therapeutic care, with $68.6 \%$ of those showing normal values for BP. There was a difference in the prevalence of hypertension according to gender and age, with males showing the highest values (M: $43.0 \%$ and F: $26.0 \%$ ) as well as $>65$-years-old people (76.2\%). Multivariate analysis (HJ-Biplot) has allowed relating the increased prevalence of hypertension with some risk factors, mainly sex, age, body mass index (BMI), lifestyle (such as smoking habits and physical activity) or disease (such as diabetes or dyslipidemia).

Conclusion: The results from the present study suggest, in comparison with other studies from Portuguese and world-wide population, the existence of a positive evolution towards reduction of the prevalence of hypertension, following an increased control, despite differences between populational subgroups, with inherent risk factors.

Keywords: Hypertension prevalence; Portuguese population; Multivariate analysis; Risk factor. 